\title{
Comportamiento de tres genotipos de uchuva (Physalis peruviana L.) bajo diferentes sistemas de poda
}

\section{Behavior of three cape gooseberry (Physalis peruviana L.) genotypes under different pruning systems}

HERNANDO CRIOLLO ${ }^{1,4}$

TULIO CÉSAR LAGOS ${ }^{1}$

GERHARD FISCHER ${ }^{2}$

LILIANA MORA ${ }^{3}$

LADY ZAMUDI0 ${ }^{3}$

Planta de uchuva en fructificación.

Foto: G. Fischer

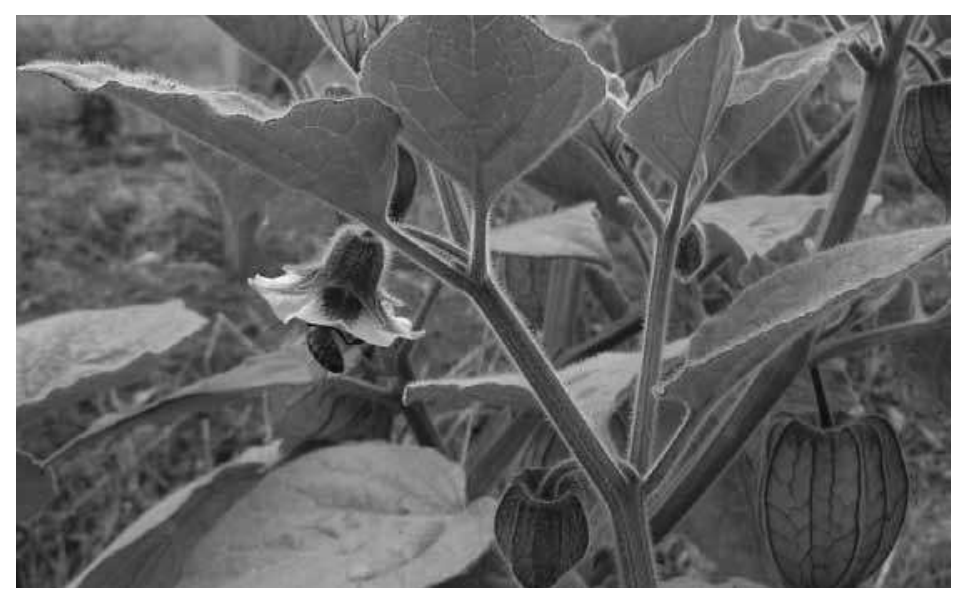

\section{RESUMEN}

Como segundo fruto de exportación en Colombia, el cultivo de uchuva tiene un gran potencial, pero hay escasez de trabajos específicos de poda de la planta en el país orientados a lograr mejores rendimientos y calidad de los frutos. Este estudio se realizó en el Centro Experimental de Botana, Universidad de Nariño (Pasto, Colombia), con el objeto de evaluar el comportamiento de tres genotipos de uchuva manejados con diferente número de ramas, en condiciones de invernadero. Se utilizó un diseño de bloques completamente al azar, con arreglo trifactorial, con cuatro repeticiones. El primer factor correspondió a tres genotipos de uchuva: Kenia, Silvania y Regional Nariño, el segundo al número de ramas primarias (3 y 4) y el tercer factor a las ramas secundarias productivas en cada una de las ramas primarias (4 y 8). Las ramas secundarias se despuntaron cuando alcanzaron un total de 25 yemas florales (nudos). Se evaluaron variables relacionadas con los variables del rendimiento y el rajado de los frutos. Los genotipos Silvania y Kenia superaron el Regional Nariño en peso fresco $(7,22$ y $5,99 \mathrm{~g} /$ fruto), diámetro $(32,18$ y $22,12 \mathrm{~mm} /$ fruto) y rendimiento $(3,67$ y $2,92 \mathrm{~kg} /$ planta, respectivamente). Mientras que el peso fresco del fruto no fue influenciado por el número de ramas, el diámetro del fruto se redujo con el aumento de las ramas secundarias. Los mayores porcentajes de rajado de frutos los presentaron 'Silvania' y 'Kenia' (8,9\% y 8,1\%, respectivamente); en 'Silvania' se redujo el rajado con el aumento de las ramas primarias.

Facultad de Ciencias Agrícolas, Grupo Producción de Frutales Andinos, Universidad de Nariño, Pasto (Colombia).

Facultad de Ciencias Agrarias, Departamento de Agronomía, Universidad Nacional de Colombia, Bogotá (Colombia). Facultad de Ciencias Agrícolas, Universidad de Nariño, Pasto (Colombia).

Autor para correspondencia. hcriollo@udenar.edu.co 
Palabras clave adicionales: ramas productivas, rendimiento, frutos, invernadero.

\section{ABSTRACT}

As the second export fruit from Colombia, cape gooseberry cultivation has vast potential, but no specific study on the pruning of this plant that is orientated toward better yields and fruit quality exists in the country. This study was carried out at the Experimental Center of Botana, University of Nariño (Pasto, Colombia) in order to evaluate the behavior of three cape gooseberry ecotypes, handled with a different number of branches, under greenhouse conditions. The statistical design was completely randomized blocks in a three-factorial arrangement with four replications. The first factor accounted for the three cape gooseberry genotypes: Kenya, Silvania and Regional Nariño; the second for the number of primary branches (3 and 4) and the third factor for the number of secondary productive branches inserted in each of the primary branches (4 and 8). The secondary branches were pinched when they reached a total of 25 floral buds (nodes). A set of variables related to the fruit, such as diameter, fresh weight, cracked fruit and yield per plant, was evaluated. The Sylvania and Kenya genotypes exceeded the Regional Nariño fruit for fresh weight (7.22 and $5.99 \mathrm{~g}$ ), fruit diameter $(32.18$ and $22.12 \mathrm{~mm}$ ) and plant yield (3.67 and $2.92 \mathrm{~kg}$, respectively). While the fresh fruit weight was not influenced by the number of branches, the fruit diameter decreased with increasing numbers of secondary branches. The higher fruit cracking percentages were seen in 'Sylvania' and 'Kenya' (8.9\% and $8.1 \%$, respectively); however, in 'Sylvania', the fruit cracking decreased with increasing numbers of primary branches.

Additional key words: reproductive branches, yield, fruits, greenhouse.

La uchuva (Physalis peruviana L.) es una especie originaria de la zona andina y en Colombia el segundo fruto de exportación (Fischer et al., 2007). En el país, los cultivos comerciales se localizan en los departamentos de Boyacá, Antioquia, Cundinamarca, Nariño, Norte de Santander, Tolima y Cauca, en 2012 se contaba con 749 ha en producción comercial y rendimientos que oscilan entre 8 y 19,9 t ha-1 (Agronet, 2014).

La uchuva presenta frutos que se forman y permanecen dentro del cáliz durante todo su desarrollo (Legge, 1974). Estos son redondos (1,25 a $2,50 \mathrm{~cm}, 4$ a $10 \mathrm{~g}$ ), de color amarillos y contienen entre 150 y 300 semillas, necesitan entre
60 y 80 d para madurar (Fischer et al., 2011) y son altamente nutracéuticos por su alto contenido en antioxidantes (vitaminas A, B, C, E y $\mathrm{K}_{1}$ ), ácidos grasos poliinsaturados, fitoesteroles y minerales esenciales (Puente et al., 2011). El cáliz tiene una consistencia apergaminada y presenta un crecimiento constante desde la época de formación floral hasta los 39 días de desarrollo; siendo este, siempre más largo que ancho (Mazorra et al., 2006).

De acuerdo con Gordillo et al. (2004), una de las grandes limitantes del cultivo es la tendencia al rajado de los frutos, lo cual puede representar pérdidas de más del 30\% totales de frutos 
comerciables, ya que se favorece la aparición de hongos y reducción de su potencial de almacenamiento. Fischer (2005) reporta que la uchuva, en sus estados iniciales de producción o en cultivos manejados con podas, es más susceptible al rajado del fruto; por esta razón, es recomendable eliminar las primeras flores.

Trabajos realizados por Torres et al. (2004) y Cooman et al. (2005) atribuyen el rajado del fruto de la uchuva a problemas relacionados con variaciones hídricas y problemas nutricionales de $\mathrm{Ca}, \mathrm{B}$ y $\mathrm{Cu}$; sin embargo, el efecto varietal no se ha tenido en cuenta y es necesario clarificarlo. En Colombia existe una gran variedad de genotipos silvestres y ecotipos reintroducidos de África (Trillos et al., 2008), pero el más cultivado corresponde al ecotipo Colombia o Silvania, por su origen y alto contenido de azúcares y mejor coloración (Mazorra et al., 2006).

El tallo principal de la uchuva que crece vegetativamente se bifurca a una altura de más o menos 0,25 m, entre el octavo a doceavo nudo, dando origen a las ramas secundarias que son altamente productivas, las ramas secundarias pueden elongarse hasta $1 \mathrm{~m}$ sin perder el vigor, con una distancia entre nudos de aproximadamente $6,5 \mathrm{~cm}$ (Fischer, 1995). En cada nudo se desarrolla una hoja, un botón floral y después de cierto tiempo se desarrolla una rama terciaria (Medina, 1991), especialmente en los nudos basales de las ramas reproductivas. Las plantas de uchuva poseen un hábito de crecimiento indeterminado, y el desarrollo de nuevas ramas, hojas, flores y frutos ocurre simultáneamente (Fischer et al., 2011).

La poda es una operación que permite manejar el crecimiento y regular el rendimiento de la planta y es usado para formar una silueta específica, adaptar la planta a una espaldera o estimular el desarrollo de ramas laterales reproductivas (Davenport, 2006). Esta práctica modifica la forma de la planta, influyendo en el número, tamaño, ángulo de inserción y la orientación de las ramas, con el fin de mejorar la estructura que debe soportar la carga de los frutos (Casierra-Posada y Fischer, 2012). Con esto se regula la capacidad vegetativa $y$ reproductiva de la planta que, según Arjona y Santinoni (2007), depende genéticamente de la especie y variedad, y es modificada por las condiciones ambientales y el manejo del fruticultor. La poda reduce el crecimiento total de la planta por lo cual es una práctica esencial en la cual el tamaño de la planta controla la producción de los frutos (Myers, 2003). Según el estado fenológico de la planta y del cultivo se distingue entre poda de plantación, formación, fructificación, mantenimiento, en verde, en seco y de rejuvenecimiento (Arjona y Santinoni, 2007). En este contexto, con la poda de fructificación se asegura un número suficiente de ramas que dan inicio a las estructuras reproductivas (Casierra-Posada y Fischer, 2012).

Teniendo en cuenta el potencial del cultivo de la uchuva y su carácter promisorio en los mercados internacionales, se consideró evaluar la productividad y calidad de los frutos en los genotipos Kenia, Silvania y Regional Nariño, sometidos a diferentes tipos de poda, dejando una cantidad diferente de ramas primarias y secundarias.

\section{MATERIALES Y MÉTODOS}

Localización. El presente trabajo se realizó bajo condiciones de invernadero con cubierta plástica, en el Centro Experimental Botana de la Universidad de Nariño, municipio de Pasto (Colombia), a 77018'8" W y 1¹0'4" N, a una altitud de $2.820 \mathrm{msnm}$, con $1.500 \mathrm{~h}$ de brillo solar promedio anual. La temperatura promedio en el invernadero fue de $16^{\circ} \mathrm{C}$ con una humedad relativa del $72 \%$.

Diseño experimental. Se trabajó con un diseño experimental de bloques completos al azar con arreglo trifactorial. Los factores correspondieron a factor 1: genotipos de uchuva (Kenia, 
Silvania y Regional Nariño); factor 2: número de ramas primarias (3 y 4 ramas) y factor 3: número de ramas secundarias por rama primaria (4 y 8 ramas). Por tanto se evaluaron 12 tratamientos, cada uno con cuatro repeticiones, para un total de 48 unidades experimentales, cada una compuesta por 4 plantas.

Manejo del cultivo. Las plantas se obtuvieron mediante enraizamiento de esquejes en sustratos suelo-arena; el trasplante en el invernadero se hizo a una distancia de 1,0 m entre surcos y 1,3 m entre plantas, en un suelo previamente preparado y con adición de abono orgánico enriquecido con abono químico 15-15-15 en una proporción de 10:1 y en una cantidad equivalente a $5 \mathrm{t} \mathrm{ha}^{-1}$. Las plantas se mantuvieron con 3 y 4 ramas primarias (nacen basalmente y son vegetativas), según el tratamiento, eliminando las sobrantes y manteniéndolas erguidas mediante tutorado. Cuando las ramas primarias alcanzaron $1 \mathrm{~m}$ de longitud, se despuntaron para inducir la emisión y mayor desarrollo de ramas secundarias, dejando 4 y 8 ramas secundarias según el tratamiento. El crecimiento de estas ramas se mantuvo, junto con las ramas terciarias formadas en cada una, hasta obtener 25 frutos/rama (incluidas las terciarias), época en que se despuntaron todas las ramas, manteniendo siempre constante el número de frutos/ rama. Al cultivo se le suministro el riego por goteo, se realizaron fertirrigaciones quincenales y los controles sanitarios requeridos.
Variables evaluadas. A partir de muestras aleatorias de 20 frutos por unidad experimental y en aquellos que cumplieron con el índice de madurez 3 de Icontec (1999), se evaluaron semanalmente el peso fresco de fruto sin cáliz (con una balanza de precisión de 0,001 g), el diámetro ecuatorial del fruto (con un calibrador tipo Rey) y el porcentaje de frutos rajados (incluyendo, según Fischer [2005], el rajado superficial ["cracking"] y profundo ["splitting"] y rendimiento [kg/planta]).

Análisis estadístico. Los datos se sometieron a análisis de varianza (Anova) y pruebas de comparación de medias de Tukey con un nivel de confianza del 95\%. Además, se realizó un análisis de regresión entre la variable número de frutos/planta originada en cada uno de los tratamientos y el rendimiento/planta.

\section{RESULTADOS Y DISCUSIÓN}

\section{Peso fresco del fruto}

El genotipo Silvania (7,22 g) fue estadísticamente superior a Kenia $(5,99 \mathrm{~g})$ y a Regional Nariño que tuvo el menor peso fresco (4,77 g) (tabla 1). Como interacción se presentó que el genotipo Kenia desarrolló frutos de menor peso $(4,86 \mathrm{~g})$ con 3 ramas primarias y 8 secundarias, mientras 'Regional Nariño' produjo frutos con un peso de 5,50 g en este tratamiento. 'Silvania' no presentó diferencias estadísticas con respecto al número de ramas; este

Tabla 1. Peso fresco (g) del fruto de tres genotipos de uchuva manejados con diferentes sistemas de poda.

\begin{tabular}{|l|c|c|c|c|c|}
\multirow{2}{*}{ Genotipo } & \multicolumn{2}{|c|}{3 ramas primarias } & \multicolumn{2}{c|}{ 4 ramas primarias } & Promedio \\
\cline { 2 - 6 } & 4 ramas secundarias & 8 ramas secundarias & 4 ramas secundarias & 8 ramas secundarias & \\
\hline Reg. Nariño & $4,59 \mathrm{~B}^{1} \mathrm{a}^{2}$ & $5,50 \mathrm{ABa}$ & $4,45 \mathrm{Ba}$ & $4,55 \mathrm{Ba}$ & $4,77 \mathrm{C}$ \\
\hline Kenia & $6,41 \mathrm{ABab}$ & $4,86 \mathrm{Bb}$ & $7,06 \mathrm{Aa}$ & $5,63 \mathrm{ABa}$ & $5,99 \mathrm{~B}$ \\
\hline Silvania & $7,10 \mathrm{Aa}$ & $7,31 \mathrm{Aa}$ & $6,98 \mathrm{Aa}$ & $7,50 \mathrm{Aa}$ & $7,22 \mathrm{~A}$ \\
\hline Promedio & $6,03 \mathrm{a}$ & $5,89 \mathrm{a}$ & $6,16 \mathrm{a}$ & $5,89 \mathrm{a}$ & 6,00 \\
\hline
\end{tabular}

Promedios con letras distintas indican diferencia significativa según la prueba de Tukey $(P \leq 0,05) .{ }^{1}$ Letras mayúsculas para comparaciones en sentido vertical (entre genotipos); ${ }^{2}$ Letras minúsculas para comparaciones en sentido horizontal (entre número de ramas). 
mismo genotipo tendió a formar frutos de mayor peso $(7,50 \mathrm{~g})$ en plantas con 4 ramas primarias y 8 secundarias, posiblemente una combinación que determina la ramificación ideal para alcanzar el mayor potencial de vertedero en los frutos de este genotipo (Fischer et al., 2012). La maximización de las zonas productoras de asimilados (área foliar) junto con el adecuado número de los vertederos (frutos) mediante una poda planificada, permitirá maximizar el número de frutos con mejor calidad y almacenar reservas para épocas de altos requerimientos (Davenport, 2006).

En el caso de la uchuva, el número de ramas cumple un papel importante, ya que según lo reportado por Fischer et al. (2008) en plantas de uchuva de 60 días desde el trasplante, la parte basal (5-20 cm sobre la ramificación) de las dos ramas reproductivas (secundarias) almacenan en peso seco 5,0\% almidón y 4,1\% sacarosa, mientras el tallo vegetativo primario en su base almacena $6,4 \%$ almidón y 5,3\% sacarosa, fuentes importantes para el llenado de frutos, diferentes de las hojas (Minchin y Thorpe, 1996).

El ecotipo Silvania, demuestra su potencial productivo y vigorosidad expresada en la capacidad de mantener un mayor peso de frutos, aun cuando se incremente de 4 a 8 el número de ramas secundarias, siendo este ecotipo producto de sucesivas selecciones (por tamaño y número de frutos) realizadas en las zonas productoras del país. Posiblemente, el alto potencial de 'Silvania' puede ser atribuido a una eficiente captación de radiación solar mediante la organización adecuada de un amplia área foliar y la optimización de sus procesos fotosintéticos, distribución y asignación de la materia seca (Marcelis, 1992; Iglesias et al., 2003).

La organización y el número de ramas es de suma importancia para la producción de esta especie; Muniz et al. (2009) evaluaron uchuvas bajo condiciones de campo abierto en Lages, SC (Brasil) y encontraron una clara superioridad del sistema en $\mathrm{X}$ (con 4 ramas principales) sobre los sistemas en espaldera simple o en $\mathrm{V}$, referente al peso fresco del fruto y la productividad.

\section{Diámetro de frutos}

Además del mayor diámetro $(P \leq 0,05)$ de los frutos del genotipo Silvania, se encontraron diferencias significativas en la interacción genotipos por número de ramas secundarias (tabla 2), destacando los frutos más grandes de 'Silvania', creciendo con 8 ramas secundarias, independiente del número de ramas primarias. En comparación, dejando 4 ramas secundarias por planta, los dos genotipos, Silvania y Kenia, superaron significativamente $(P \leq 0,05)$ el diámetro de los frutos del 'Regional Nariño' (tabla 2). Probablemente, con 4 ramas secundarias entró más luz a la planta asegurando frutos de buen tamaño (Marsal et al., 2006); además, se presenta una menor competencia por nutrientes y asimilados (Arjona y Santinoni, 2007), en comparación con

Tabla 2. Diámetro (mm) del fruto de tres genotipos de uchuva manejados con diferentes sistemas de poda.

\begin{tabular}{|l|c|c|c|c|c|}
\multirow{2}{*}{ Genotipo } & \multicolumn{2}{c}{3 ramas primarias } & \multicolumn{2}{c|}{ 4 ramas primarias } & \multirow{2}{*}{ Promedio } \\
\cline { 2 - 6 } & 4 ramas secundarias & 8 ramas secundarias & 4 ramas secundarias & 8 ramas secundarias & \\
\hline Reg. Nariño & $21,56 \mathrm{~B}^{1} \mathrm{a}^{2}$ & $20,19 \mathrm{Ba}$ & $18,90 \mathrm{Ba}$ & $19,01 \mathrm{Ba}$ & $19,91 \mathrm{~B}$ \\
\hline Kenia & $24,54 \mathrm{Aa}$ & $19,52 \mathrm{Bb}$ & $23,77 \mathrm{Aa}$ & $20,66 \mathrm{ABb}$ & $22,12 \mathrm{~B}$ \\
\hline Silvania & $23,53 \mathrm{ABa}$ & $23,19 \mathrm{Aa}$ & $23,01 \mathrm{Aa}$ & $22,98 \mathrm{Aa}$ & $23,18 \mathrm{~A}$ \\
\hline Promedio & $23,21 \mathrm{a}$ & $20,96 \mathrm{~b}$ & $21,89 \mathrm{ab}$ & $20,88 \mathrm{~b}$ & 21,74 \\
\hline
\end{tabular}

Promedios con letras distintas indican diferencia significativa según la prueba de Tukey $(P \leq 0,05)$. 'Letras mayúsculas para comparaciones en sentido vertical (entre genotipos); ${ }^{2}$ Letras minúsculas para comparaciones en sentido horizontal (entre número de ramas). 
las plantas de 8 ramas secundarias situación que se manifestó especialmente en las plantas con 3 ramas primarias (tabla 2).

Esta poda permite regular la cantidad y el tamaño de frutos producidos a través de la regulación del número y de la longitud de las ramas secundarias, mejorando la partición de fotoasimilados mediante la cantidad de los vertederos (frutos) y su localización sobre las ramas (Fischer et al., 2012).

\section{Porcentaje de frutos rajados}

El análisis de varianza mostró diferencias estadísticas entre genotipos y en la interacción entre genotipos y número de ramas primarias. Con 3 ramas primarias, los genotipos Silvania y Kenia presentaron mayores porcentajes de rajado (12,5\% y $7,50 \%)$, mientras que 'Regional Nariño' mostró un mejor comportamiento con un menor valor de rajado (tabla 3), lo cual coincide con Fischer (2005) cuando afirma que la incidencia del cuarteamiento del fruto varía ampliamente entre las diferentes especies y variedades.

Por su parte, la disminución de rajado en los genotipos Silvania y Regional Nariño cuando aumentó el número de ramas primarias y así la carga de frutos, está en concordancia con los resultados de Ayala (1995) que encontró una disminución considerable del agrietamiento cuando se dejaron 980 frutos/planta con $25,2 \mathrm{~kg}$ de frutos rajados/ ha, comparado con 179,6 $\mathrm{kg} \mathrm{ha}^{-1}$ rajados dejando solamente 320 frutos/planta. Según Peet (1992), la alta carga de frutos en la planta puede reducir el riesgo del rajado porque la concentración de los sólidos solubles en el fruto disminuye.

El porcentaje más alto $(P \leq 0,05)$ del rajado observado en 'Kenia' y 'Silvania', posiblemente se debe al mayor tamaño y acumulación de peso fresco de estos frutos, comparado con los del 'Regional Nariño'; esto coincide con estudios de Gordillo et al. (2004) en la uchuva 'Colombia', en los cuales el porcentaje de frutos rajados aumentó cuando hubo un menor número y un mayor tamaño de los frutos en la planta. A parte del reducido número de frutos en la planta, esta condición también es genética, ya que según Fischer y Lüdders (1997), el tamaño del fruto está ligado a factores genéticos responsables del número de células del ovario formadas antes de la antesis, lo cual determina la velocidad de crecimiento, el tamaño y la forma final del fruto.

Es importante resaltar la respuesta del genotipo 'Silvania', manejado con 4 ramas primarias, en cuanto a la reducción del porcentaje de rajado alcanzado con 3 ramas. Al aumentar el número de ramas, la planta posee un mayor volumen transpiratorio, incremento de la masa celular y una mejor distribución hídrica (Marsal et al., 2006), por lo cual se supone que esta situación reduce el riesgo de ruptura de la superficie del fruto por la disminución de la presión ejercida sobre las paredes celulares a nivel de pericarpio. Así se genera

Tabla 3. Porcentaje de rajado de frutos de tres genotipos de uchuva manejados con diferente número de ramas primarias.

\begin{tabular}{|l|c|c|c|}
\multicolumn{1}{|c|}{ Genotipo } & 3 ramas primarias & 4 ramas primarias & Promedio \\
\hline Reg. Nariño & $3,62 \mathrm{~B}^{1} \mathrm{a}^{2}$ & $2,50 \mathrm{Ba}$ & $3,06 \mathrm{~B}$ \\
\hline Kenia & $7,50 \mathrm{Aa}$ & $8,62 \mathrm{Aa}$ & $8,06 \mathrm{~A}$ \\
\hline Silvania & $12,50 \mathrm{Aa}$ & $5,25 \mathrm{Ab}$ & $8,88 \mathrm{~A}$ \\
\hline Promedio & $7,87 \mathrm{a}$ & $5,41 \mathrm{a}$ & 6,66 \\
\hline
\end{tabular}

Promedios con letras distintas minúsculas indican diferencia significativa según la prueba de Tukey $(P \leq 0,05)$; ${ }^{1}$ Letras mayúsculas para comparaciones en sentido vertical (entre genotipos); ' Letras minúsculas para comparaciones en sentido horizontal (entre número de ramas). 
que la planta de uchuva con 4 ramas primarias tenga un mejor equilibrio fisiológico entre la formación de flores y frutos junto con el crecimiento e inserción de nuevas ramas (Mathieu et al., 2008), esto se traduce en una mayor producción de frutos y un menor porcentaje de rajamiento.

\section{Rendimiento por planta}

El rendimiento por planta registrado durante una época (pico) de cosecha determinó que 'Silvania' superó significativamente a los genotipos Regional Nariño y Kenia (tabla 4). Cuando se evaluó el efecto de las ramas primarias, los genotipos tuvieron comportamientos productivos similares, sin efecto significativo; cuando se evaluó el efecto de las ramas secundarias, se presentaron diferencias significativas entre genotipos, siendo el 'Regional' el menos productivo, tanto con 4 $(1,51 \mathrm{~kg} / \mathrm{pl}$.) como con 8 ramas $(3,33 \mathrm{~kg} / \mathrm{pl}$.), con respecto al genotipo 'Silvania' $(2,36 \mathrm{~kg} / \mathrm{pl}$. y 4,98 $\mathrm{kg} /$ pl., respectivamente).

El rendimiento de los genotipos Kenia y Silvania mejoró notablemente con el incremento de las ramas primarias, mientras que los tres genotipos presentaron mayores rendimientos al incrementar el número de ramas secundarias de 4 a 8 , aumentando casi en un $100 \%$ la la producción (tabla 4), lo que coincide con lo reportado en lichi (Litchichinensis) donde el aumento del número de los flujos (ramas) reproductivos indujo un mayor rendimiento de frutos (Chang y Lin, 2008). A pesar del alto número de ramas (hasta 32 ramas secundarias) en las uchuvas se mantuvo un equilibrio fisiológico entre su crecimiento vegetativo y el rendimiento de frutos garantizando una máxima producción.

El análisis de regresión permitió establecer el comportamiento productivo de cada uno de los genotipos frente a los tratamientos analizados. Con 3 ramas primarias y 4 secundarias (300 frutos/planta) los genotipos Silvania y Kenia mostraron producciones similares y superiores al del genotipo Regional Nariño (figura 1). Con el incremento del número de frutos/planta, el genotipo Silvania mostró una respuesta superior a Kenia, aumentando significativamente su rendimiento y demostrando su adaptación; el genotipo Kenia mostró un incremento menos significativo en su rendimiento al aumentar el número de frutos/planta a 600, aunque este incremento fue superior al de 'Regional Nariño'. Este resultado demostró que con el aumento del número de ramas secundarias, más que con un mayor número de las primarias, se puede aprovechar mejor el potencial productivo de la uchuva hasta alcanzar su máximo potencial, nivel que, posiblemente, todavía no se ha logrado (Fischer et al., 2007).

En la medida que aumentó el número de frutos por planta se incrementó su rendimiento, un resultado que no coincide con el estudio de Ayala (1995), quien formó plantas de uchuva bajo invernadero de plástico con 1 rama primaria y 4 ramas secundarias, variando el número de ramas terciarias y la cantidad de frutos dejados en estas, obteniendo entre 320 y 980 frutos/planta con rendimientos de 10,4 y $3,46 \mathrm{tha}^{-1}$, respectivamente. Esta drástica reducción del rendimien-

Tabla 4. Rendimiento (kg/planta) de tres genotipos de uchuva manejados con diferentes sistemas de poda.

\begin{tabular}{|l|c|c|c|c|c|}
\multicolumn{1}{|c|}{ Genotipo } & 3 ramas primarias & 4 ramas primarias & 4 ramas secundarias & 8 ramas secundarias & Promedio \\
\hline Reg. Nariño & $2,24 \mathrm{~A}^{1} \mathrm{a}^{2}$ & $2,60 \mathrm{Aa}$ & $1,51 \mathrm{Bb}$ & $3,33 \mathrm{Ba}$ & $2,42 \mathrm{~B}$ \\
\hline Kenia & $2,32 \mathrm{Ab}$ & $3,52 \mathrm{Aa}$ & $2,28 \mathrm{ABb}$ & $3,56 \mathrm{ABa}$ & $2,92 \mathrm{~B}$ \\
\hline Silvania & $3,13 \mathrm{Ab}$ & $4,22 \mathrm{Aa}$ & $2,36 \mathrm{Ab}$ & $4,98 \mathrm{Aa}$ & $3,67 \mathrm{~A}$ \\
\hline
\end{tabular}

Promedios con letras distintas indican diferencia significativa según la prueba de Tukey $(P \leq 0,05) .{ }^{1}$ Letras mayúsculas para comparaciones en sentido vertical (entre genotipos); ${ }^{2}$ Letras minúsculas para comparaciones en sentido horizontal (entre número de ramas). 


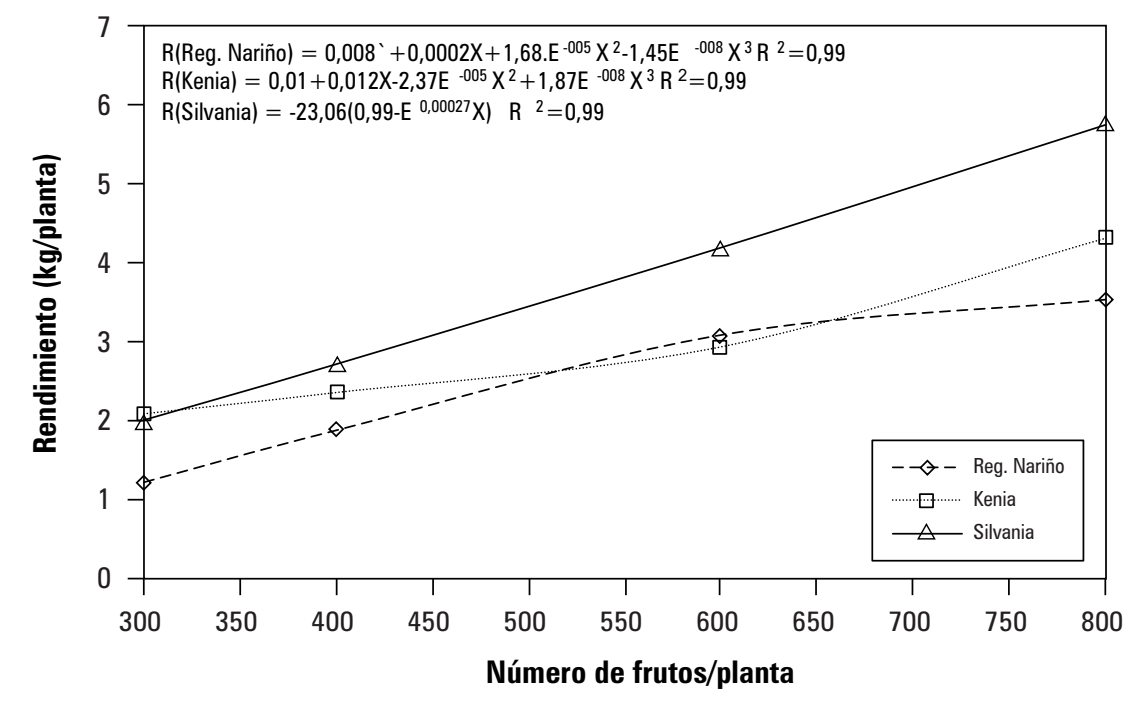

Figura 1. Respuesta de la producción de genotipos de uchuva, según el número de frutos manejados con base en los diferentes tipos de poda ( 3 ramas primarias y 4 secundarias $=300$ frutos/ planta; 4 ramas primarias y 4 secundarias $=400$ frutos/planta; 3 ramas primarias y 8 secundarias $=600$ frutos/planta, 4 ramas primarias y 8 secundarias $=800$ frutos $/$ planta).

to como lo reporta Ayala (1995), fue por el bajo porcentaje de frutos tipo extra y primera, en las plantas que se condujeron con 980 frutos/planta.

Las condiciones climáticas pueden modificar los resultados de producción en las diferentes épocas y sistemas de plantación, conducción y poda como encontraron Muniz et al. (2009) en Lages (SC) y Lima et al. (2010) en Pelotas (RS, Brasil).

\section{CONCLUSIONES}

El incremento en el número de ramas secundarias fue más importante que el incremento de ramas primarias en la generación de un mayor rendimiento de las plantas de uchuva.

Con un menor número de ramas primarias aumentó la incidencia del rajado del fruto, especialmente en el genotipo Silvania, debido, posiblemente, a una sobrecarga en los flujos de agua y fotosintetizados hacia los frutos.

El genotipo Silvania superó a Kenia y Regional Nariño, presentando mayor peso fresco de fruto y por consiguiente un aumento en el rendimiento por planta, por lo que este genotipo es apto para futuros programas de selección y fitomejoramiento.

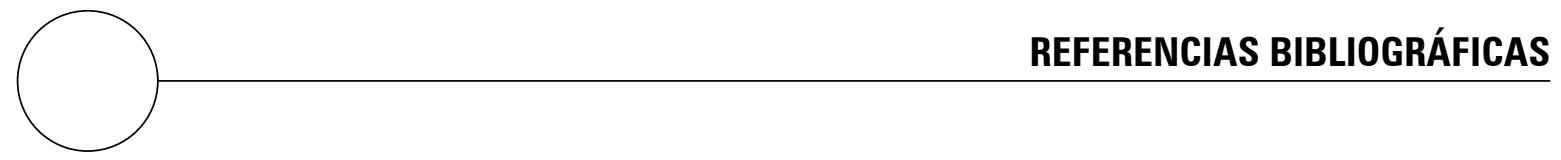

Agronet. 2014. Uchuva. En: www.agronet.gov.co; consulta: marzo de 2014.
Arjona, C. y L.A. Santinoni. 2007. Poda de árboles frutales. pp. 243-282. En: Sozzi, G.O. (ed.). Árboles 
frutales, ecofisiología, cultivo y aprovechamiento. Universidad de Buenos Aires, Buenos Aires.

Ayala, C. 1995. Evaluacion de tres densidades de siembra y tres sistemas de poda en el cultivo de uchuva (Physalis peruviana Linnaeus), ecotipo Colombia, bajo invernadero. Tesis de maestría. Facultad de Agronomía, Universidad Nacional de Colombia, Bogotá.

Casierra-Posada, F. y G. Fischer. 2012. Poda de árboles frutales. pp. 169-185. En: Fischer, G. (ed.). Manual para el cultivo de frutales en el trópico. Produmedios, Bogotá.

Cooman, A., C. Torres y G. Fischer. 2005. Determinación de las causas del rajado del fruto de uchuva (Physalis peruviana) bajo cubierta. II. Efecto de la oferta de Ca, B y Cu. Agron. Colomb. 23, 1-10.

Davenport, T.L. 2006. Pruning strategies to maximize tropical mango production from the time of planting to restoration of old orchards. HortScience 41,544-548.

Chang, J.-C. y T.-S. Lin. 2008. Fruit yield and quality as related to flushes of bearing shoots in litchi. J. Amer. Soc. Hort. Sci. 133, 284-289.

Fischer, G. 2005. El problema del rajado del fruto de uchuva y su posible control. pp. 55-82. En: Fischer, G., D. Miranda, W. Piedrahita y J. Romero (eds.). Avances en cultivo, poscosecha y exportación de la uchuva (Physalis peruviana L.) en Colombia. Unibiblos, Universidad Nacional de Colombia, Bogotá

Fischer, G. 1995. Effect of root zone temperature and tropical altitude on the growth, development and fruit quality of the cape gooseberry (Physalis peruviana L.). Tesis de doctorado. Humboldt-Universität Berlin, Berlín.

Fischer, G., P.J. Almanza-Merchán y F. Ramírez. 2012. Source-sink relationships in fruit species. A review. Rev. Colomb. Cienc. Hortic. 6, 238-253.

Fischer, G., F. Beran y Ch. Ulrichs. 2008. Partitioning of non-structural carbohydrates in the fruiting cape gooseberry (Physalis peruviana L.) plant. En: Memorias Tropentag. 22-24 Oct. 2008. University of Hohenheim, Stuttgart, Alemania.

Fischer, G.; G. Ebert y P. Lüdders. 2007. Production, seeds and carbohydrate contents of cape gooseberry (Physalis peruviana L.) fruits grown at two contrasting Colombian altitudes. J. Appl. Bot. Food Qual. 81(1), 29-35.

Fischer, G., A. Herrera y P.J. Almanza. 2011. Cape gooseberry (Physalis peruviana L.). pp. 374-396.
En: Yahia, E.M. (ed.) Postharvest biology and technology of tropical and subtropical fruits. Vol. 2. Acai to citrus. Woodhead Publishing, Cambridge, UK.

Fischer, G. y P. Lüdders. 1997. Developmental changes of carbohydrates in cape gooseberry (Physalis peruviana $\mathrm{L}$.) fruits in relation to the calyx and the leaves. Agron. Colomb. 14(2), 95-107.

Gordillo, O., G. Fischer y R. Guerrero. 2004. Efecto del riego y de la fertilización sobre la incidencia del rajado en frutos de uchuva (Physalis peruviana L.) en la zona de Silvania (Cundinamarca). Agron. Colomb. 22(1), 53-61.

Icontec. 1999. Frutas frescas. Uchuva. Especificaciones. Norma Técnica Colombiana NTC 4580. Instituto Colombiana de Normas Técnicas, Bogotá.

Iglesias, D.J., I. Lliso, F. Tadeo y M. Talón. 2003. Regulation of photosynthesis through source-sink balance in citrus is mediated by carbohydrate content in leaves. Plant Physiol. 116, 563-57.

Legge, A.P. 1974. Notes on the history, cultivation and uses of Physalis peruviana L. J. Royal Hort. Sci. 99(7), 310-314.

Lima, C.S.M., M.A. Gonçalves, Z.F.P Tomaz, A.R. Rufato y J.C. Fachinello. 2010. Sistemas de tutoramento e épocas de transplante de Physalis. Ciência Rural 40(12), 2472-2479.

Marcelis, L. 1992. The dynamic of growth and dry matter distribution in cucumber. Ann. Bot. 69, 487-492.

Marsal, J., G. López, M. Mata y J. Girona. 2006. Branch removal and defruiting for the amelioration of water stress effects on fruit growth during stage III of peach fruit development. Sci. Hortic. 108, 55-60.

Mathieu, A., P.H. Cournede, D. Barthélémy y P. de Reffye. 2008. Rhythms and alternating patterns in plants as emergent properties of a model of interaction between development and functioning. Ann. Bot. 101, 1233-1242.

Mazorra, M., A. Quintana, D. Miranda, G. Fischer y M. Chaparro. 2006. Aspectos anatómicos de la formación y crecimiento del fruto de uchuva Physalis peruviana (Solanaceae). Acta Biol. Colomb. 11(1), 69-81.

Medina, M.E. 1991. El cultivo de la uchuva tipo exportación. Agric. Trop. 28(2), 55-64.

Minchin, P.E.H. y M.R. Thorpe. 1996. What determines carbon partitioning between competing sinks? J. Expl. Bot. 47, 1293-1296. 
Muniz, J., A.A. Kretzschmar, L. Rufato, T.R. Pelizza, T. Marchim A.E. Duarte, A.P.F. Lima y F. Garanhani. 2011. Sistemas de condução para o cultivo de Physalis no planalto catarinense. Rev. Bras. Frutic. 33(3), 830-838.

Myers, S.C. 2003. Training and pruning principles. pp. 339-345. En: Baugher, A. y S. Singha (eds.). Concise enciclopedia of temperate tree fruit. Food Products Press, Nueva York, NY.

Puente, L.A., S.A. Pinto-Muñoz, E.S. Castro y M. Cortés. 2011. Physalis peruviana Linnaeus, the multiple properties of a highly functional fruit: A review. Food Res. Intl. 44, 1733-1740.

Torres, C., A. Cooman y G. Fischer. 2004. Determinación de las causas del rajado del fruto de uchuva (Physalis peruviana) bajo cubierta. I. Efecto de la variación del balance hídrico. Agron. Colomb. 22, 140-146.

Trillos, O., J. Cotes, C. Medina, M. Lobo y A. Navas. 2008. Caracterización morfológica de cuarenta y seis accesiones de uchuva (Physalis peruviana L.), en Antioquia (Colombia). Rev. Bras. Frutic. 30(3), 708-715 